\title{
III. \\ Polypöse Wucherungen im äusseren Gehörgang, bedingt durch ein Sarkom der Schädelbasis.
}

\author{
Von \\ Dr. Stetter in Königsberg in $\mathrm{Pl}^{2}$. \\ Docent der Chirurgie und Ohrenheilkunde.
}

(Mit 2 Abbildungen.)

(Von der Redaction übernommen am 1. April 1892.)

Von mehrfachen Gesichtspunkten aus halte ich den folgenden Fall der Veröffentlichung werth. Erstens wegen der mit dem Gehirnleiden verbundenen Lähmungserscheinungen im Gebiete aller betroffenen Nerven, d. b. aller vom 3. bis zum 12. Paare, ferner wegen der colossalen Knochenzerstörungen an der Basis cranii, und drittens wegen der Krankheitserscheinungen von Seiten des Obres.

Patient, 31 Jahre alt, gab an, schon seit seiner Militärzeit häufig an Kopfschmerzen gelitten za baben, welche besonders in der linken Seite auftraten und stets auch mit schmerzhaften Empfindungen im linken Ohre verbunden waren. Im Herbst 1890 stellte sich wieder mit gleichzeitigem Schmerz in der linken Kopfhälfte ein mit geringem Ausfluss aus dem Ohre verbundenes Ohrleiden ein, über welches aber genauere Angaben nicht gemacht werden können. Etwa 6 Wochen vor Aufnahıme in die Klinik (9. Juli a. pr.) erkrankte Patient plötzlich unter allgemeinen fieberhaften Symptomen mit Husten, so dass er bettlägerig wurde. Im Auschluss an diese Erkrankung steigerten sich die Kopfschmerzen, der Ohrenfluss wurde bedentender, nnd es traten Beschwerden beim Sprechen und Schlucken anf; die Stimme wurde heiser, der Husten sehr erschwert, und bei dem Genusse von festen Speisen musste Patient fortwährend trinken, damit das Hinunterschlucken, welches immer nur schwer und langsam gelang, besser von Statten ginge; zuweilen missglitekte ihm jeder Schlingversuch, und es erfolgte dann Erbrechen. Der Ausfuss ans dem Ohre nahm beständig zu und wurde übelriechend.

Status praesens. Bei der Untersuchung des Patienten, der entschieden den Eindruck eines sehwer kranken, deprimirten Menschen macht, fällt sofort die Veränderung der Sprache auf; sie ist tonarm, ohne direct tonlos zu sein, sie schlägt sehr leicht ins Falset über, 
oder sie klingt für einen Laut als Doppelstimme, derartig, dass eine tiefe und eine hohe Stimme za gleicher Zeit anklingen. Der Kranke spricht mit sichtbarer Anstrengung der Exspirationsmuskeln. Nach tiefer Inspiration, den Oberkörper und namentlich den Kopf etwas nach vorniiber geneigt, wie zum Husten und Expectoriren, setzt der Patient mit einer stossweisen Exspiration zu dem zu sprechenden Worte an, aber schon nach $2-3$ Worten muss er wieder von Nenem tief inspiriren; kurz, er spricht unter dem akustischen Eindruck einer ausserordentlichen Luftverschwendung. Tritt zufällig wälırend der Untersuchung, oder weil der Larynx durch Sondiren gereizt wird, Husten auf, so quält er den Kranken furchtbar. Der Husten ist tonarm, rasch verhauchend, und der Kranke setzt immer wieder rasch von Neuem zu einem Hustenstosse an, so dass dieser Husten mehr krampfartig erseheint.

Die laryngoskopische Untersuchung ergiebt Folgendes: Die Epiglottis ist etwas stärker injicirt, in geringstem Grade sattelförmig gekrümmt, steht gegen die Larynxöffnung geneigt und verdeckt demgemäss zum Theil den Eingang in den Keblkopf. Obgleich die Epiglottis zunächst nur auf die Wrisberg'schen, bezw. Santorinischen Knorpelflächen der Schleimhaut des Arytaenoidknorpels einen Blick gestattet, bemerkt man doch eine wesentliche Veränderung. Der rechte Arytaenoid, bezw. Wrisberg'sche Knorpel hat anscheinend den linken aus der Mittellinie nach links verdrängt. Es besteht keine Regio interarytaenoidea, sondern die verdickte, etwas geschwellte Schleimhant der rechten Cartilago arytaenoidea steht etwas vor und über der der linken. Blitzartig zuckt der rechte Arytaenoidknorpel, wenn Patient nicht spricht und nicht athmet, auf und seheint sich zu erbeben nnd vorzudrängen, als wollte er den linken Arytaenoidknorpel noch mehr nach hinten und links zur Seite schieben. Lässt man den Kranken phoniren, so erhebt sich die Epiglottis so weit, dass ein Einblick in den Larynx gestattet ist. Man sieht dann das in seiner vorderen Hälfte erbeblich verschmälerte linke Stimm. band der Mittellinie genähert, während der Athmung sowohl, als auch während der Phonation absolut stillstehen, es ist sehnig-weiss, scharfrandig und säbelförmig concav gekrümmt. An der hinteren Fläche quillt die subchondrale-Schleimbaut etwas vor. Bei Phonationsversuchen wird das linke Stimmband wie ein sehlaffes Segel mit groben Schwingungen nach oben und seitlich gegen die Kehlkopfswand geblasen. Das rechte $\mathrm{stimmband}$ ist in toto breiter, von normaler Farbe, steht etwas schräg nach aussen in normaler Stellung und bewegt sich respiratorisch und phonatorisch; in der Ruhestellung springt der Proc. vocalis etwas vor. Bei der Phonation wird nur die hinterste Hälfte des Stimmbandes, speciell der Proc. vocalis nach links hinibergelegt, auch wird das ganze Stimmband aus seiner schrägen Stellung etwas in die Medianrichtung gebracht, aber trotzdem weder die Glottis cartilaginea, noch weniger die Glottis ligamentosa dadurch geschlossen. Es bleibt während der Phonation ein halb ovaler Spalt, und es seheint dabei das linke Stimmband, im Vergleich zum rechten, schräg nach aussen zu stehen. In voller Spannung 
scheint sich aber auch das rechte Stimmband während der Phonation nicht zu befinden; je höher der Kranke zu phoniren versucht, desto schlaffer und gröber sind die Vibrationen reehterseits. Sebr auffallend ist ferner eine $\mathrm{Niveandifferenz} z$ wischenden beiden Stimmbändern. Das rechte, beweglichere, steht zweifellos um mehrere Linien tiefer als das linke, und zwar auch sehon in der Ruhestellung des Kehlkopfes. Diese Niveandifferenz seheint von hinten nach vorn etwas zuzunehmen, ist jedoch während der Phonation nicht sicher festzustellen. Sie scheint anch bei wiederholter Untersuchung nicht die gleiche zu sein und beträgt zuweilen sicherlich 2-3 Mm., zuweilen auch nur $1 \mathrm{Mm}$. Die Sensibilität der Schleimhaut ist intact, ein Verschlucken findet nicht statt.

Das hintere Drittel des linken Gehörgangs wird ausgefullt von einer fleischrothen, mit dünnem, serös-eitrigem, übelriechendem Secret bedeckten Granulationsmasse, welche bei vorsichtiger trockener Reinigung leicht blutet. Auf dem Secret liegt ein schwach pulsirender Lichtreflex. Vom Trommelfell ist nichts zu sehen. Die Gehörsfunction ist sowohl Seitens der Luft-, wie auch Seitens der Knochenleitung ganz erloschen, der Stimmgabelton lateralisirt für hohe wie tiefe Töne von der Mittellinie des Schädels, sowie vom linken Scheitelbein und vom linken Warzenfortsatz nach der rechten Seite. Eine Facialislähmung ist nicht vorhanden, auch ist an den Augenbewegungen nichts Abnormes nachzuweisen, und anch die Sehschärfe ist normal.

Danach war die Diagnose zu stellen auf: Otitis media purulentasin. cum granulationibus, Lähmung des linken N. acusticus, Lähmung des linken Stimmbandes und des Oesophagus.

Verlauf. Da keine Fiebererscheinungen bestanden, so glaubte ich vorläufig von einer Trepanation des Schädels Abstand nehmen zu dürfen, da es wenig wahrscheinlich war, dass die Lähmungen durch eine Abscedirung bedingt wurden, die ihren Weg vom Mittelohre aus entlang der Basis cranii nahm. Ich behandelte daher unter genauer Beobachtung aller übrigen Krankheitssymptome zuerst die Granulationswucherung im linken Ohre mit Kauterisationen, jedoch ohne sichtbaren Erfolg. Da dem Kranken das Schlucken von Tag zu Tag schwerer wurde, nahm Herr Prof. Schreiber, den ich bat, den Patienten auf die Lähmungserscheinungen hin genau zu untersuchen, am 20. Juli eine Sondirung des Oesophagus vor. Dabei fiel auf, dass selbst die dicksten Sonden sebr leicht einzuführen waren, und dass Patient augenscheinlich keine Gefühlswahrnehmung in Oesophagus hatte. Nach Einfiihrung verschieden dieker Sonden in versehiedene Tiefe dentete Patient stets auf die Frage, wo er die Sonde fühle, nach dem Pharynx und behauptete bestimmt, tiefer unten keine Empendung zu haben. Am 11. August trat eine linksseitige Facialisparese ein. Die Sensibilität war dabei ibberall normal, ebenso der Geschmack und der Geruch. Der Kranke klagte uber eine Schwäche im linken Arm, die linke Schulter stand bedeutend tiefer als die rechte. Die Pupillen reagirten prompt, jedoch war die 
linke kleiner als die rechte. Am 12. August stellten sich Schmerzen dicht unterhalb des linken Proc. mastoid. ein, der ebenso wie die seitlichen Fortsätze der oberen Halswirbel auf Druck sehr schmerzhaft war. Die Haut über dem Warzenfortsatz war geröthet und zeigte eine leichte ödematöse Anschwellung.

Dieser Befund liess nunmehr einen operativen Eingriff als durchaus nothwendig erscheiren. Ich schritt jedoch nicht zur Trepanation des Proc. mastoid., sondern eröffnete die Schädelhöhle weiter nach hinten im Bereiche der linken Hinterhauptsgrube, da die Symptome von Seiten der Gehirnnerven dafür sprachen, dass es sich um einen intracraniellen fortsch reitenden Entziudungsprocess handele, nicht um einen abgekapselten Eiterherd im Proc. mastoideus. In der Annahme daher, dass eine eitrige basilare Meningitis vorliegen möchte, legte ich die Schädelöffnung hinter dem Proc. mastoid. an. Die freigelegte harte Hirnhaut zeigte keinen eitrigen Belag, sah etwas dunkler aus, als normal, und pulsirte nicht. Nach ihrer Spaltung. entleerte sich viel dunkles venöses Blut, aber kein Eiter.

Der Wundverlauf war ein durchaus normaler, die Lähmungserscheinungen änderten sich jedoch gar nicht, dagegen nahmen die Schmerzen im Nacken zu, und es traten auch solche in den Armen auf. Die Palpation ergab längs des Plex. cervicalis und brachialis linkerseits deutliche Druckempfindlichkeit. Am 24. August zeigte sich bei der Abnahme des Verbandes eine leichte ödematöse Schwellung, vom Proc. mastoid. beginnend und vor dem M. sternocleidomastoid. nach abwärts ziehend. Die ganze Stelle war schmerzhaft. Patient klagte ausserdem über heftige Kopfschmerzen. - Urin normal; im Sputum keine Bacillen.

Am 7. September wurde zum ersten Male eine Parese des linken N. abducens bemerkt, denn der Patient konnte das linke Auge nicht mehr über die Mittellinie nach aussen hin bewegen. Die Sehschärfe blieb aber normal. Die Untersuchung des Augenhintergrundes ergab die Zeichen einer Staungspapille, besonders starke Schlängelung und Erweiterung der Venen.

In den nächsten Wochen änderte sich der Zustand wenig, die Temperatur blieb zwischen 37 und $38^{\circ}$, Patient sprach immer noch sehr heiser, nahm aber, da ihm das Schlucken anscheinend von Tag zn Tag schwerer wurde, nur sehr wenig zu sich. Das Ohr wurde täglich gereinigt, jedoch stand ich bei dem elenden Allgemeinbefinden des Patienten selbstverständlich davon $a b$, durch eine eingreifende Operation die immer weiter wuchernden Granulationen zu entfernen, um so mehr, als die successive Mitbetheiligung der an der Gehirnbasis verlaufenden Gehirnnerven, zusammengenommen mit dem Allgemeinbefinden des Patienten, die Diagnose eines Hirntumors zur Sicherheit erhob. Unter Zunahme der allgemeinen Schwäche, jedoch ohne Hinzutritt weiterer Lähmungserscheinungen und anch ohne merkliche Abnahme der geistigen Functionen, trat am 9. October der Tod ein, nachdem sich in den letzten Tagen noch eine beiderseitige Bronchitis zu den übrigen Leiden hinzugesellt hatte.

Die am 10. October von Herrn Dr. Askanazi, Assistent am 
Kgl. pathologischen Institute, gemachte Section ergab folgenden Befund :

Die linksseitige Fossa conchae ist mit schmatziggrünem Secret bedeckt, welches die Mündung des äusseren Gehörgangs erfüllt. Das Schädeldach, ziemlich dick, symmetriseh gebaut. Dura gespannt, mit mässiger Gefässfüllung. Im Sinus longitudinalis wenige Tropfen fliüssigen Blutes. Die Innenfläche der Dura mater glatt, das subarachnoideale Bindegewebe namentlich hinten durch reichlichen Liquor getruibt. Die Pia mater ist bis in die feinsten Ramificationen mit Blut gefüllt und trägt hinten einige stärker gefillte Venenstämme. Etwa $1 \mathrm{Cm}$. von der Fissura magna, der Wurzel der ersten Stirnwindung entsprechend, findet sich auf der Arachnoidea, resp. den ans derselben hervorgegangenen, leicht verkalkten Pacchioni'schen Granulationsknöpfehen eine circa fünfpfennigstückgrosse blasige Erhebung, die aus mehreren erbsengrossen Abtheilungen besteht (Cysticercus, wie die sofort vorgenommene Untersuchung des Inhaltes ergab).

Die Hirnnerven der rechten Seite erscheinen frei, ebenso die linksseitigen bis zum 6. Paare, während die tieferen zunächst nicht zu Tage treten. Das 6., 7. und 8. Paar reissen bei Abhebung des Cerebellums durch. An der linken Seite, im Bereiche der hinteren Hälfte der Schläfenbeinpyramide, befindet sich eine Vorwölbung, uiber welche die harte Hirnhaut prall gespannt ist, und es zeigt sich hier eine Verwachsung der Basalfläche des Kleinhirns mit den darunter liegenden Theilen. Nach Durchschneidung dieser Verwachsung und Abhebung der Dura mater wird eine Höhle sichtbar mit einem graugrünlichen, übelriechenden, aus weichen Klumpen zusammengesetzten Inhalt. An mehreren Punkten stösst die Sonde auf rauhen Knochen. Nach Herausnahme des Gehirns übersieht man die Höhle deutlich. Ein Theil der hinteren Pyramidenwand und die linke Hälfte des Clivus Blumenbachii sind zerstört. Mit der Sonde fühlt man eine grössere Zahl blossliegender, zerstörter Knochentheile; der eingeführte Finger gelangt unter der Haut bis zum Proc. mastoid. In der Höhle befindet sich jauchiger, missfarbiger Eiter, die Wände derselben sind zum grössten Theil mit blassem Granulationsgewebe bedeckt. In den vordersten Abschnitten des Sinus transvers. sin. findet sich ein weicher röthlicher Thrombus, der nur in der medianen Partie leicht missfarbig erscheint. Im Sinus transvers. dext. befindet sich etwas frisch geronnenes Blut. Nach Entfernung der Weichtheile des Schädels gelingt es, an vielen Stellen des linken Schläfen- und Hinterhauptbeins die Sonde zwischen cariös zerstörten Knochentheilen hindurchzuführen. Auch die Nerven vom 9. Paare an verlieren sich in der Jauchenhöhle.

In der linken Lunge bestand eine eitrige Bronchitis, auf dem Durchschnitt markirten sich vereinzelte graurothe bronchopneumonisclse Herde. Es fand sich ferner ein Milztumor, Kalkinfarete der Nieren und eine versprengte Nebenniere an der rechten Nierenrinde.

Die mikroskopische Untersuchnng ergab, dass ein Sarkom der Schädelbasis die ausgedehnten Knochenzerstörungen hervor- 
gerufen hatte, und dass dasselbe in hohem Grade jauchig zerfallen war. Die Partie des Kleinhirns, welehe mit der Dura fest verwachsen war, wurde mit Letzterer herausgeschnitten und in Alkohol gebärtet. Dabei fiel auf, dass die adhärirende Stelle der Kleinhirnhemisphäre sehr derb war. Mikroskopisch zeigte sich der betreffende Theil des Kleinhirns vollständig in das Gewebe eines grosszelligen alveolären Rundzellensarkoms umgewandelt, welches in der Dura

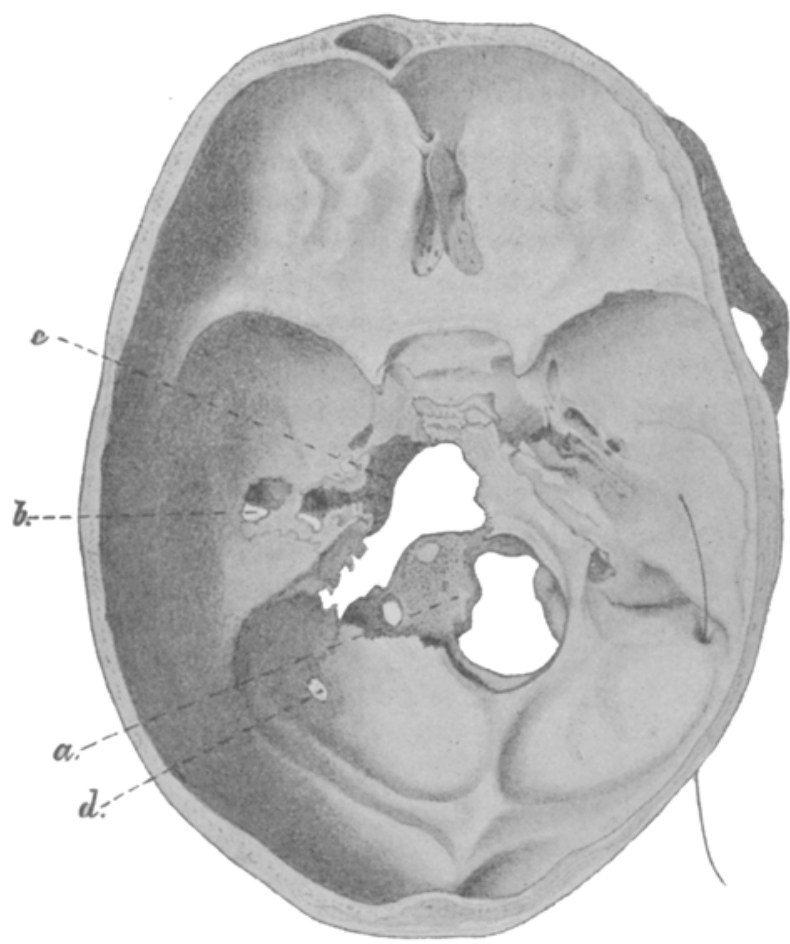

Fig. 1.

$a$ Die cariöse Fossa glenoidalis des L. Halswirbels. $\quad b$ Cariöser Defect der Fossa glenoidalis des Unterkiefers, $c$ Unterkiefer. $d$ Trepanationsöffnung.

noch kleine Nester bildete. In einer Region docnmentirt sich das Durchwuchern der Geschwulst von der Innenwand der Jauchenhöhle durch die Dura hindurch in das Sarkomgewebe des Kleinhirns ohne Unterbrechung. Auch in der entzündlichen Infiltration der zottigen Innenfläche der Höhle lassen sich Geschwulstzellen erkennen.

Die Zerstörungen an der Sehädelbasis erstrecken sich, wie aus den beigegebenen Figuren ersichtlich ist, auf das Hinterhauptsbein, das Felsenbein, das Keilbein, auf den ersten Halswirbel und den Gelenkkopf des Unterkiefers. Ebenso zieht sich eine oberflächliche 
cariöse Zerstörung des Knochens durch den ganzen Sulcus transvers. sin. bis zur Protaber, oceip. int. Von den genannten Knochen fehit ein Theil ganz, andere Theile zeigen oberflächliche und tiefere cariöse Zerstörungen. Es fehlen die vorderen zwei Drittel der linken Umrandung des grossen Hinterhauptsloches, so dass die eariöse linksseitige Gelenkfläche des Atlas ganz zu übersehen ist, es fehlt die linke Hälfte des Clivus Blumenbachii, so dass ein freier Einblick in die Höble des Keilbeinkörpers gestattet ist, es fehlt ferner die mediale und basale Hälfte der Pars petrosa des Sehläfenbeins, sowie der ganze knöcherne und knorpelige Gehörgang, so dass zwischen der vorderen Fläche des Proc. mastoid. und der hinteren Fläche des Unterkieferhalses ein leerer

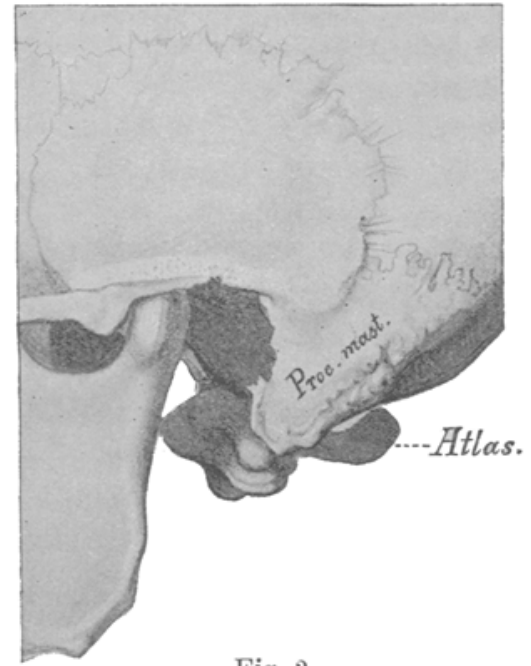

Fig. 2. Raum besteht, wie aus Fig. 2 ersiehtlich ist. Von den für die Nervenbahnen besonders wichtigen Theilen sind zerstört:

1. das Foramen spinosum (dessen hintere Begrenzung fehlt);

2. der Meatus audit. int.;

3. das Foramen jugulare;

4. das Foramen condyl.ant.

Es sind also die Durchtrittsstellen folgender Gehirnnerven zerstört, diese selbst von der Geschwulst umwachsen und zum grossen Theilo in sie aufgegangen:

1. der N. petrosus superfacialis minor;

2. der N. acusticus und $N$. facialis ;

3. der N. glossopharyngeus, vagus und accessorius Willisii;

4. der N. hypoglossus.

Der $N$. facialis und acusticns waren an ihrer Austrittsstelle in dem Winkel zwischen der Medulla oblongata und dem vorderen Rande des Kleinhirus von der Gesehwulst umwachsen, ebenso der N. abducens vor seinem Eintritt in die Fissura orbitalis superior.

Von welcher Stelle das Sarkom seinen Ausgang genommen hatte, liess sich bei der vorliegenden hochgradigen Zerstörung natürlich nicht mehr nachweisen. Die anamnestischen Angaben lassen jedoch die Vermuthung gerechtfertigt erscheinen, dass der Beginn der Erkrankung im Ohre stattgefunden hat, da sich neben den zuerst auftretenden Kopfschmerzen sehr bald auch ein Ausfuss aus dem Ohre einstellte. Leider waren von dem Patienten keine irgend zuverJässigen Angaben über die Mitbetheiligung des Gehörs im Anfang des Leidens zu erhalten.

Von einer Behandlung dieser bösartigen Geschwillste der Schädel- 
basis kann wohl kaum die Rede sein, selbst wenn es gelingt, die genaue mikroskopische Diagnose schon früh zo stellen dureh die Untersuchung der in dem äusseren Gehörgang nach Zerstörung des Trommelfells hervorgewucherten Geschwulsttheile, deren Entfernung von vornherein bei sonst gutem Allgemeinbefinden des Patienten jeder Ohrenarzt, oder überhaupt wohl jeder Arzt ohne Weiteres vornehmen wïrde. Ganz abgesehen davon, dass es sich in Fällen, wie der vorliegende, nicht um eine abgegrenzte, sondern am eine infiltrirte (v. Bergmann), ohne erkennbare Grenze in die gesunde Hirnsubstanz ubergehende Geschwulst handelt, so ist eine Entfernung auf chirnrgischem Wege von der Schädelbasis nicht angängig; denn nicht nur infolge ihrer Ausbreitung, ihrer leichten, wohl sicheren Recidivfähigkeit, sondern auch infolge ihres Sitzes gehören die Sarkome der Schädelbasis zu denen, die einer operativen Behandlung überhaupt nicht zugänglich sind.

Von hohem Interesse scheint mir aber noch der Umstand zu sein, dass und wie der Patient seinen Kopf, allerdings vorwiegend nach der linken Seite geneigt, bis zu seinem Tode uberhaupt halten, wenn auch langsam und unter Schmerzen, so doch sogar ausgiebig drehen und sowohl nach vorn, wie nach hinten beugen konnte, da doch der ganze linke Condylus des Hinterhanptbeins fehlte, also alle Bewegungen des Kopfes gegen den Atlas nur in dem einen, rechtea, Gelenk ausgeführt werden konnten.

Erwähnenswerth ist endlich noch das Vorhandensein eines Cawalis mastoid. auf der rechten Seite. Derselbe stellte einen an der Basis des Proc. mastoid. verlaufenden Kanal dar, dessen äussere Mündung genall am hinteren Rande der Basis des Zitzenfortsatzes lag, dessen innere Oeffnnng in die Fossa sigmoidea mindete, und dessen Lumen $4 \mathrm{Mm}$. betrug. Hyrtl erwähnt hier ein zuweilen fehlendes Foramen mastoideum. 